\title{
Improving perinatal outcomes: lessons learned from a low middle-income public hospital: An observational study from North India
}

\author{
Anita Kaul ${ }^{1}$, Smriti Prasad ${ }^{2}$, Vanamail Perumal ${ }^{3}$, Rachna Gupta ${ }^{4}$, and Akshatha Sharma ${ }^{5}$ \\ ${ }^{1}$ Indraprastha Apollo Hospitals \\ ${ }^{2}$ APOLLO CENTRE FOR FETAL MEDICINE \\ ${ }^{3}$ AIIMS \\ ${ }^{4}$ Sunehri Devi Hospital \\ ${ }^{5}$ Indraprastha Apollo Hospital
}

May 6, 2020

\begin{abstract}
Evaluate the impact of third trimester ultrasound in identifying, small for gestational age (SGA) growth restricted (FGR) and appropriately grown fetuses (AGA). Analyse their outcomes ,ascertain the main causes of perinatal death and lastly compare ultrasound intervention to standard antenatal care . Design Prospective observational study Setting Secondary care, government hospital Population Antenatal women Methods Based on estimated fetal weight by scan, patients were categorized into FGR $<3$, SGA $>3$ and $<10$ and AGA $>10$ centile. Perinatal outcomes and deaths were analysed by category. Health Ministry data was extracted for standard care outcomes and compared to study group. Main Outcomes Perinatal deaths and causes,composite neonatal morbidity ,severe adverse outcomes Results Number of births in study and standard care was 1817 and 15,427 respectively. Detection rate by ultrasound for FGR/SGA fetuses was $28.5 \%$. This contained $44.8 \%$ (13/29) of all perinatal deaths. Neonatal deaths, composite neonatal morbidity, serious adverse perinatal outcomes were significantly more in FGR compared to AGA. Standard care group had twice as many stillbirths RR 2.32(1.3-4.1)and early neonatal deaths (RR2.5 (1.18-5.34) . Majority of perinatal deaths $(17 / 29 ; 58.6 \%)$ in study group were the direct result of failure to recognize antepartum/ intrapartum risk factors, leading to delayed delivery. In addition $(3 / 12 ; 25 \%)$, women reported late after decreased fetal movements and ( $3 / 1225 \%$ )were post dated. Ill-equipped nursery contributed to neonatal mortality in $(10 / 17 ; 58.8 \%)$ cases. Conclusion Perinatal outcomes will not improve by ultrasound identification of small fetuses unless concomitantly, obstetric protocols and patient awareness programmes are not improved.
\end{abstract}

\section{Objective}

Evaluate the impact of third trimester ultrasound in identifying, small for gestational age (SGA) growth restricted (FGR) and appropriately grown fetuses (AGA).Analyse their outcomes ,ascertain the main causes of perinatal death and lastly compare ultrasound intervention to standard antenatal care .

\section{Design}

Prospective observational study

\section{Setting}

Secondary care, government hospital

\section{Population}


Antenatal women

\section{Methods}

Based on estimated fetal weight by scan, patients were categorized into FGR $<$ 3rd, SGA $>3$ and $<10$ th and AGA $>10$ th centile. Perinatal outcomes and deaths were analysed by category. Health Ministry data was extracted for standard care outcomes and compared to study group.

\section{Main Outcomes}

Perinatal deaths and causes, composite neonatal morbidity, severe adverse outcomes

\section{Results}

Number of births in study and standard care was 1817 and 15,427 respectively. Detection rate by ultrasound for FGR/SGA fetuses was $28.5 \%$ This contained $44.8 \%$ of all perinatal deaths. Neonatal deaths, composite neonatal morbidity, serious adverse perinatal outcomes were significantly more in FGR compared to AGA. Standard care group had twice as many stillbirths RR 2.32(1.3-4.1)and early neonatal deaths (RR2.5 (1.18$5.34)$.

Majority of perinatal deaths $(17 / 29 ; 58.6 \%)$ were the direct result of failure to recognize antepartum/ intrapartum risk factors, leading to delayed delivery. In addition $(3 / 12 ; 25 \%)$, women reported late after decreased fetal movements and ( $3 / 1225 \%)$ were post dated.

\section{Conclusion}

Perinatal outcomes will not improve by ultrasound identification of small fetuses unless concomitantly, obstetric protocols ,infrastructure and patient awareness programmes are not improved.

\section{Funding}

There was no source of financial support or funding.

\section{Keywords}

Stillbirth,perinatal death, third trimester ultrasound,fetal growth restriction,low middle income country, antenatal care

\section{Introduction}

An estimated 2.6 million third-trimester stillbirths occurred in 2015, and almost all were in low and middleincome countries (LMIC)

1. Approximately 590,000 (22.7\%) of these stillbirths occurred in India, representing the most significant numbers of stillbirths globally. ${ }^{2}$

While, a multiplicity of factors contribute to stillbirth, over half are related to placental dysfunction, many of these associated with impaired fetal growth ${ }^{3}$ This suggests that antenatal detection of fetal growth restriction (FGR), should lead to an overall decrease in stillbirths with the majority being preventable. ${ }^{4-6}$

Not only does India have the most significant number of small for gestational age (SGA) births of any country, at 12.8 million, but the overall percentage of SGA within the country is also extremely high $(46.9 \%)$ 7

Universal third trimester ultrasound has been shown to triple the detection rate of SGA infant ${ }^{8}$

Currently, the Indian government only mandates a mid-trimester ultrasound ${ }^{9}$

We proposed that a third-trimester ultrasound may better identify the growth-restricted fetus.

Our primary hypothesis was stillbirth, along with neonatal morbidity and mortality will be lower in the cohort of women undergoing third-trimester ultrasound in a specialized obstetric scanning clinic when compared to 
women undergoing standard antenatal care in the same hospital. The health statistics for the latter will be derived from data submitted to the Ministry of Health and Family Welfare of India, ${ }^{10}$ during the same study period.

Secondary analysis would be performed on all perinatal deaths in the study group to ascertain preventable causes. We hoped to achieve the target set by the India Newborn Action Plan (INAP), ${ }^{11}$ the first national stillbirth-prevention target set at achieving $<10$ stillbirths per 1,000 births by 2030

\section{Methods}

This was a prospective observational study.

\section{Setting}

This pilot project was part of a Public-Private healthcare partnership between Delhi government (study hospital) and a corporate hospital (providing specialist fetal medicine sonographers). Data were prospectively collected between September 2017 and September 2019 at a 100-bed government secondary care hospital conducting approximately 6100 deliveries per year. This hospital caters to lower socio-economic population and has emergency obstetric services available at all times, a Blood Bank, and a Neonatal Intensive Care Unit (NICU).High risk pregnancies are referred early to tertiary hospitals for antenatal care or anticipated very small fetuses $<1000 \mathrm{~g}$ are in-utero transferred to a tertiary hospital.

\section{Recruitment / randomisation}

Inclusion criteria included low -risk women with singleton intrauterine pregnancies, A hospital radiology unit, consisting of two radiologists, performed all clinically indicated scans (roughly 800 obstetric scans/month, at varying gestational age).

A parallel study unit was set up to perform routine third-trimester scans three days each week and first trimester or anomaly scans two days each week. Initially, women were referred to this unit at varying gestational ages as that was the system being followed in the hospital. Eventually we managed to get patients scheduled for scans at 11-13 weeks, 18-20 weeks, and between 32.0-37.6 weeks. The majority of women who presented for the third-trimester scans came directly from the community, brought in by ASHA workers (Accredited Social Health Activist). ${ }^{12}$ These women were if they had an early pregnancy dating report.

Primary Outcomes - Severe Adverse Perinatal Events

Four primary outcomes, chosen based on relevance and practicality

2) included:

a) Neonatal seizures, defined as clonic movements which cannot be stopped by holding the limb, occurring on two or more occasions before 72 hours of age;

b) Assisted ventilation for more than 24 hours via endotracheal tube, initiated within 72 hours after birth; Maternal death within 48 hours of giving birth.

\section{Secondary Outcomes}

Four secondary outcomes were assessed:

1) Emergency caesarean section due to fetal distress;

2) Interval (days) to delivery once FGR identified

3) Malpresentations and congenital anomalies identified in the third trimester

4)Ascertainable causes of stillbirth

\section{Data Collection}


All women signed an informed consent agreeing to their participation, along with the PCPNDT (PreConception and Pre-Natal Diagnostic Techniques Act 1994) form. ${ }^{15}$

At the growth scan, the biparietal diameter (BPD), head circumference (HC), abdominal circumference $(\mathrm{AC})$, and femur length (FL) measurements were taken using standard reference planes. Estimated fetal weight (EFW) was calculated using the Hadlock formula.

The umbilical artery, middle cerebral artery (MCA), and ductus venosus pulsatility indices (PI) were recorded, along with the amniotic fluid index (AFI) according to standard protocol.

Hadlock EFW chart ${ }^{16}$ was used to calculate EFW and growth centile as these charts are the ones used locally.

If a woman had scans on multiple occasions during the third trimester, then the last scan closest to delivery, was included.

All fetuses [?] $10^{\text {th }}$ percentile were identified. Fetuses [?] $3^{\text {rd }}$ percentile were labelled as FGR and $>3^{\text {rd }}$ percentile and [?] $10^{\text {th }}$ percentile were labelled Small for Gestational Age ( SGA). Appropriately grown fetuses (AGA) were those between the $10^{\text {th }}$ and $90^{\text {th }}$ percentile, and Large for gestational age (LGA) $>$ $90^{\text {th }}$ percentile. The latter were excluded from the analysis.

The final birth weight at delivery was obtained and plotted on Fenton's gender birth weight chart ${ }^{17}$ considered as the reference chartfor assessing SGA/FGR/AGA by local neonatologists, both in the study hospital and the tertiary care hospitals.

All patients were managed according to local obstetric protocol. If fetal Doppler suggested impending demise, only then were the obstetric units alerted by a phone call. Delivery was performed according to obstetric indications.

Outcomes were collected from the delivery records or by a telephonic interview if the woman did not deliver in the study hospital. Neonatal admission data were taken from the hospital records or from the patient.

All study stillbirths had a pre-structured questionnaire filled and an internal review to ascertain cause and assign a CODAC $\operatorname{code}^{18}$ (Causes of Death and Associated Conditions )

All the scans were performed on GE Voluson S8 ultrasound system (GE Healthcare; Zipf, Austria). Data was entered into an obstetric database Astraia $\mathrm{GmbH}$ from which the appropriate parameters were extracted.

\section{Data collected from the Ministry of Health for Standard care}

All hospitals are required to submit data to the Ministry of Health and Family Welfare for several health indicators. Data were extracted from these records for the number of deliveries, the number of vaginal deliveries, the number of caesarean sections done at night (this was taken as proxy for intrapartum caesarean sections although actual number would have been higher as the intrapartum caesarean sections during the day are not reported separately), the number of stillbirths (which included both antenatal and intrapartum deaths occurring after 28 week gestation, quantified together, as the data is reported as a composite), the number of early and late neonatal deaths and, finally, the number of maternal deaths occurring during the same time period at the same hospital as the study duration.

\section{Power Calculation}

Due to logistics, we did not achieve the desired sample size of 2700 but rather $67.3 \%$ of the required sample size. However we calculated the power of the study in the post-hoc analysis where we reached $94 \%$ and $81 \%$ power for the stillbirth rate and early neonatal death rate respectively. Since both these measures were part of our primary outcomes, our study is adequately powered with the sample size of 1817

\section{Statistical Analysis}


The sample size was calculated based on a stillbirth rate in Delhi $(2.9 \%)$ obtained from an earlier study. Presuming that through ultrasound, the stillbirth rate can be reduced by $30 \%$ a sample size of 2700 would have statistical significance with $80 \%$ power at a $5 \%$ level of significance. We analysed the data in the study cohort according to the defined subcategories of FGR, SGA and AGA. Next, we compared the outcome variables of study cohort to official data submitted to Delhi government over the same duration using STATA software version 12.0(2011) Continuous variables were tested for normality assumption using KolmogorovSmirnov test. We calculated descriptive statistics for normally distributed data and compared the mean values using (ANOVA)/Student's t-independent test, as appropriate. We presented categorical variables as

frequency and percent values and compared using Chi-square/Fishers exact test. Unadjusted risk ratio and $95 \%$ confidence limits were calculated to identify risk variables for various outcomes. For all statistical tests, a two-sided probability of $\mathrm{P}<0.05$ was considered for statistical significance.

\section{Results}

Progress of the study by different stages is shown in Flowchart S1

\section{Baseline maternal and demographic characteristics are presented in Table S2}

Of the 1817 study subjects, the majority were young and primigravida, with on average education up to middle school level. Overall ,fetuses less [?] $10^{\text {th }}$ centile accounted for $18 \%$ (329/1817) of the antenatal scans. A total of $48.59 \%(883 / 1817)$ of fetuses were $<10^{\text {th }}$ centile based on actual birthweight (Fenton chart). Further 33\% (425/1817) had FGR and 25.26\% (459/1817) had SGA on actual birth weight. Antenatal scans, therefore, detected approximately $28.1 \%$ of the fetuses at $<10^{\text {th }}$ percentile.

Primary outcomes by sub-categories are presented in Table S3

The majority of the study population in all sub-categories (1336/1817: $73.52 \%$ ) had vaginal delivery.

\section{Adverse outcomes by subcategories are shown in Table 1}

Although the rates of stillbirths did not differ substantially between the sub-categories, statistically significant difference was noted for neonatal mortality. The FGR group had significantly high rate of early neonatal deaths (relative risk $(\mathrm{RR})=15.75,95 \% \mathrm{CI}: 3.56-69.58 ; \mathrm{P}<0.05)$ and deaths greater than one week $(\mathrm{RR}=$ 9.45; 95\% CI : CI $-2.57-34.74 ; \mathrm{P}<0.05)$.

The composite neonatal morbidity was also markedly higher in the FGR and SGA groups compared to the AGA group. There was one incident of neonatal seizures, and one maternal death, secondary to complications of postpartum haemorrhage.

Our adverse outcomes were mainly confined to those patients with abnormal third trimester ultrasounds.

Table 2 Comparison of the adverse outcomes between the study group and standard care

This showed that the stillbirth rate was $15.3 / 1000$, neonatal death (NND) $<1$ week was $9.7 / 1000$ and MMR was 450/100,000 in the standard care. All figures were significantly worse than in the study group. Neonatal morbidity could not be compared as that data is not submitted to the Ministry.

The table of the relevant standard care data submitted to the Ministry is seen in Table S4

Table 3 shows the stillbirths and Table 4 shows the neonatal deaths by individual case analysis in the study group to ascertain the primary etiology , assign CODAC code for retrieval in future studies, and determine where corrective measures could be instituted We noted that the majority of perinatal deaths $(17 / 29 ; 58.6 \%)$ were caused by failure to recognize antepartum and intrapartum risk factors, leading to delayed delivery. In a few cases $(3 / 12 ; 25 \%)$, it appears that women reported late for reduced perception of fetal movements or were post-dates and presented with an intrauterine demise at the hospital $3 / 12$ (25\%).There was one death due to poorly managed labour for a breech delivery. We identified 10/17 (58.8\%) cases where the neonatal facility of the hospital was ill-equipped to manage the neonate, contributing to neonatal mortality. 
It was alarming to see the number of adverse events even in the study group and it was thought necessary to review some protocols of obstetric management in the study cohort following the Royal College of Obstetrician and Gynaecologists professional guidelines ${ }^{19,20}$

\section{Review of few obstetric management protocols}

Firstly it was noted that once FGR was identified, only $28.57 \%(36 / 126)$ were delivered by 37.6 weeks. Another $22.2 \%$ were delivered post-dates( Table S5 shows Gestational Age at delivery of FGR cohort )

Secondly, there were $90 / 124(72.58 \%)$ women who had an umbilical artery PI more than $95^{\text {th }}$ centile and were undelivered beyond 37.6 weeks Out of these 25/37(67.57\%) had EFW [?] $3^{\text {rd }}$ centile and 20/28 (71.43\%)had EFW between $3^{\text {rd }}$ to [?] $10^{\text {th }}$ centile. Nearly $15 \%$ in all groups were post-dates (Table S6)

Thirdly, we identified 31 fetuses with malpresentation (breech). In primigravida women with breech presentation, over(7/26) 30\% had either vaginal births or emergency C-sections beyond 39 weeks. On follow-up telephonic enquiry, it was found that many of these patients had not understood the importance of follow up.

There were three cases identified antenatally with structural abnormalities (congenital diaphragmatic hernia, ventricular septal defect, tracheoesophageal fistula) while one heart defect was found postnatally.

We can extrapolate that similar findings would have been seen in the standard care group which may have also contributed to the high perinatal mortality seen in that group.

\section{Discussion}

Main Findings

In our cohort, the stillbirth rate was 6.6/1000, meeting the INAP target of less than 10/1000.

Antenatal scans detected nearly $28.5 \%$ of fetuses $<10^{\text {th }}$ percentile. This group comprised of $44.8 \%$ of all

The study group had significantly better outcomes than the standard care group as depicted in Table 2 .

\section{Strengths and Limitations}

Our study population is representative of low -risk antenatal women seeking routine obstetric care. Care was provided at a typical municipal non-teaching hospital. All clinically indicated ultrasounds were routinely performed in Radiology, thereby minimising bias of abnormal referrals. With nearly $99 \%$ follow-up, these findings can be used to review and advise maternity health priorities.

\section{Interpretation}

The study demonstrated that a third-trimester ultrasound at a dedicated scanning clinic could triage patients who needed increased fetal surveillance.

We detected approximately $28 \%$ fetuses with FGR, which is less than the meta-analysis, suggesting that when using EFW, third-trimester US had a detection rate of about $35 \%^{21}$ for identifying SGA infants. Our study agrees with Madden et $\mathrm{al}^{22}$ that SGA infants from low-risk, term pregnancies are at increased risk of serious neonatal morbidity.

Lindqvist et al have suggested that unidentified SGA is a common finding in perinatal deaths ${ }^{23}$ This may have accounted for the deaths that were seen in the AGA group given the high prevalence of small babies in the study group(48.59\%).

We feel that unlike the Pregnancy Outcome Prediction ${ }^{8}$ study which suggested that universal screening in the third trimester would roughly triple the detection rate of FGR to approximately $57 \%$,,in our setting without a higher threshold for labelling SGA ${ }^{24}$ or the ability to assess a decrease in growth velocity, this detection rate ${ }^{25}$ is unachievable. 
The Cochrane meta-analysis, ${ }^{26}$ IRIS study ${ }^{27}$ and First Cluster Study ${ }^{28}$ do not support a routine thirdtrimester scan, suggesting that it does not improve fetal and neonatal adverse outcomes.

The Cochrane meta-analysis found no difference in antenatal, obstetric and neonatal outcomes in ultrasound screened versus control groups. There was no association between ultrasound in late pregnancy and perinatal mortality (risk ratio (RR) 1.01, 95\% confidence interval (0.67 to 1.54 ).

The IRIS trial compared the use of routine third-trimester biometry to clinically indicated ultrasound in low-risk pregnant women. They found no significant improvement between the two groups; however, they used a multidisciplinary protocol for the detection and management of fetal growth restriction, in both the screening strategies.

The above studies are Western trials with excellent antenatal services, a robust midwifery structure, and protocolized management once a small fetus is identified.

In our setting women often receive intermittent and infrequent obstetric care, and the fundal height/palpation may not be checked consistently in the antenatal clinics. In this scenario, many small fetuses may be missed. These could. Unfortunately, even when FGR was identified, an effective intervention strategy was not instituted. The Cluster First Look Low Middle Income Trial, demonstrated this and concluded that without improvement in the quality of antenatal care, there would be limited impact in the use of routine ultrasound alone. Additionally, studies have shown ${ }^{28,30}$ identifying FGR without adequate follow up or higher quality institutional deliveries will be ineffectual in preventing stillbirths.

Studies from India have suggested the care is suboptimal at several levels ${ }^{31,32}$ At the patient level, there is failure to report decreased fetal movement, default in follow up, refusal to be hospitalised or undergo intervention, the influence of socio-cultural factors (e.g., not to report to the hospital unless spontaneous labour starts), and infrequently homebirths.

At the hospital level, causes cited included failure to manage high-risk cases, delay or error in labour management, inadequate fetal monitoring or inexperienced doctors, and the lack of equipment required for fetal surveillance in labour

Studies from Bihar ${ }^{33,34}$ evaluated population-based risk factors for stillbirth. It emphasised that the INAP implementation would not be successful unless several improvements were made in obstetric care. These included improved documentation and audit, especially if delivery was deferred; ${ }^{35}$ improved quality of care, teaching women how to monitor fetal movements Additionally, communicating test results to patients, monitoring and evaluating referral practices, and increased public-private partnerships would likely lead to decreased rates of stillbirth.

Our causes of perinatal deaths echo those of the authors cited here.

In our study cohort, patients were asked to report written findings to their obstetrician which is the routine followed in most public hospitals. If, instead, time had been taken with the patient, the attendant, or the ASHA worker, to emphasise the importance of noting their due date, tracking fetal movements, returning for scheduled follow-up visits, and ensuring they understood the process of referral to a tertiary hospital, deaths may have been prevented. Franklin et $\mathrm{al}^{36}$ examined barriers to women reaching higher centres. They found that convincing women to proceed with recommendations involves critical communication, including describing ultrasound findings, reasons for the referral, where to go in the hospital, and what to expect at the hospital.

Maternal perception of decreased fetal movements is associated with adverse pregnancy outcomes, including stillbirth ${ }^{37,38}$ Teaching patients how to monitor fetal movements is recommended in the Indian health worker guidelines for delivery of $\mathrm{care}^{39}$ However the results of our study suggest that this may not be practised consistently. It has been recommended that to strengthen the surveillance mechanism to prevent stillbirths further research is needed to understand the best methods to monitor fetal movements, and what information women should receive from their providers. ${ }^{40,41}$ 
We suggest that the ASHA workers be given more responsibility and be taught to check the fundal height in order to ensure appropriate hospital visits.

One suggestion to improve perinatal outcomes is to maintain a black book ${ }^{42}$ by the ultrasound clinic which contained the date of the follow-up scan and contact phone numbers of the patient, family ,neighbour and community health worker. A call was made to encourage a follow-up visit, once the recommended scan date had passed. The prevalence of cell phones, even in this strata, makes it possible to incorporate this intervention into current practice.

\section{Conclusions}

1. Perinatal mortality and morbidity rates will not improve unless hospitals follow protocolized obstetric management.

2. Every pregnant woman needs to be proactively taught to monitor fetal movements and be aware of her due date.

3. ASHA workers need to be instructed to measure fundal height so that community identification of a small fetus for subsequent hospital antenatal visit can be identified,

4. Separate obstetric scanning clinics are better at triaging high-risk patients but need to communicate findings to patients more effectively

5. A black book follow-up approach needs to be instituted.

6. Referrals to higher centres need to be timely so that the patients do not get lost in the system.

7Public -private partnerships need to be encouraged so that skilled private healthcare workers are used to augment the care given by government doctors with no increase in financial liability to the Government.

We demonstrated that third-trimester ultrasound could identify nearly half of the fetuses that were at increased risk of perinatal mortality and morbidity. Third-trimester scanning needs to be offered given the high prevalence of small fetuses in our population .Current professional society obstetric guidelines for fetal monitoring, the timing of induction of labour, and better intrapartum management, along with strong patient awareness programmes will significantly improve outcomes.

Acknowledgements

Dr Amita Saxena, Dr Rachna Jain, Dr Naveen ,Sister Chinggouman and all the obstetric and neonatal staff of the study hospital for their unstinting cooperation in facilitating this study.

The clinical fellows of Apollo Centre for Fetal Medicine Dr Saloni Arora ,Dr Ayesha Ahmad, Dr Aanchal Sablok ,Dr Nilesh Mhaske and Dr Keerthana Anand as well as the senior staff who contributed in data collection.

The study was supported by GE Healthcare (Zipf, Austria) by providing the Voluson S8 ultrasound device and probes for ultrasound examinations during the study period and coordinated by Dr Laura S. Schultz and her team

Astraia GmbH donated the use of the obstetric database for the study period and Mr Roland Denk and Mr Rakesh Rai gave their continuous technical support.

Dr Nirmala Agarwal President ,All India Royal College Of Obstetricians and Gynaecologists -North Zone India who through the organisation donated a computer ,printer and peripherals for the study.

Fetal Medicine Foundation India who donated computer and peripherals for the study

\section{Disclosure of Interests}

AK,RG,AS are on the Executive committee of Fetal Medicine Foundation India and the other authors have no disclosure of interests 


\section{Contribution to Authorship}

AK conceived and designed the study, collected literature, analysed and interpreted data and wrote the paper

SP collected, analysed constructed data tables, collected literature and reviewed the paper

PV analysed the data, performed the statistical analysis and critically reviewed the paper

$\mathrm{RG}$ analysed and interpreted the data and wrote the paper

AS collected, analysed and interpreted the data and wrote the paper

Ethical Approval

Ethical clearance was obtained from the Institutional Ethical Committee Order No 86(1)MS/LBSH/2016/10469 dated 24/11/2016

Funding

There was no source of financial support or funding.

\section{References}

1. Lawn JE, Blencowe H, Waiswa P, Amouzou A, Mathers C, Hogan D, et al. 1. Stillbirths: rates, risk factors, and acceleration towards 2030. The Lancet. Elsevier; 2016 Feb 6;387(10018):587-603.

2. Blencowe H, Cousens S, Jassir FB, Say L, Chou D, Mathers C, et al. National, regional, and worldwide estimates of stillbirth rates in 2015, with trends from 2000: a systematic analysis. The Lancet Global Health. Elsevier; 2016 Feb 1;4(2):e98-e108.

3. Pattinson R, Kerber K, Waiswa P, Day LT, Mussell F, Asiruddin S, et al. Perinatal mortality audit: Counting, accountability, and overcoming challenges in scaling up in low- and middle-income countries. International Journal of Gynecology and Obstetrics. John Wiley \& Sons, Ltd; 2009 Oct 1;107(Supplement):S113-22.

4. Froen JF, Cacciatore J, McClure EM, Kuti O, Jokhio AH, Islam M, et al. Stillbirths: why they matter. The Lancet. Elsevier; 2011 Apr 16;377(9774):1353-66.

5. Stanton C, Lawn JE, Rahman H, Wilczynska-Ketende K, Hill K. Stillbirth rates: delivering estimates in 190 countries. The Lancet. Elsevier; 2006 May 6;367(9521):1487-94.

6. De Reu PAOM, Oosterbaan HP, Smits LJM, Nijhuis JG. Avoidable mortality in small-for-gestational-age children in the Netherlands. Journal of Perinatal Medicine. De Gruyter; 2010 May 1;38(3):311-8.

7. Black RE. Global Prevalence of Small for Gestational Age Births. 2015 May

$5 ;: 1-8$.

8. Sovio U, R White I, Dacey A, Pasupathy D, C S Smith G Prof. Screening for fetal growth restriction with universal third trimester ultrasonography in nulliparous women in the Pregnancy Outcome Prediction (POP) study: a prospective cohort study. The Lancet. Sovio et al. Open Access article distributed under the terms of CC BY; 2015 Nov 21;386(10008):2089-97.

9. India GO. Ultrasound guidelines GOI .Available from: http://www.nrhmhp.gov.in/sites/default/files/files/Approved\%20Guidelines\%20on\%20use\%20of\%20Ultrasonography.pdf

10. Health MO. Delhi Government Available from: http://health.delhigovt.nic.in/wps/wcm/connect/DoIT_Health/health/home/ 
11. India GO. India Newborn Action Plan [2014 [Available from: https://nhm.gov.in/index4.php?lang=1\&level=0\&linkid=153\&lid=174

12. Agarwal, S., Curtis, S.L., Angeles, G. et al. The impact of India's accredited social health activist (ASHA) program on the utilization of maternity services: a nationally representative longitudinal modelling study. Hum Resour Health 17, 68 (2019). https://doi.org/10.1186/s12960-019-0402-4

13. Fetal Medicine Foundation Available from: fetalmedicine.org

14. WHO | Stillbirths. WHO World Health Organization. Available from: https://www.who.int/maternal_child_adolescent/epidemiology/stillbirth/en/

15. PCPNDT Act Available from: http://pndt.gov.in

16. Hadlock FP, Harrist RB, Sharman RS, Deter RL, Park SK. Estimation of fetal weight with the use of head, body, and femur measurements - A prospective study. YMOB. Elsevier; 1985 Feb 1;151(3):333-7.

17. Fenton TR, Kim JH. A systematic review and meta-analysis to revise the Fenton growth chart for preterm infants. BMC Pediatr. 2013;13(1):59.

18. Froen JF, Pinar H, Flenady V, Bahrin S, Charles A, Chauke L, et al. Causes of death and associated conditions (Codac) - a utilitarian approach to the classification of perinatal deaths. BMC Pregnancy and Childbirth. 31st ed. BioMed Central; 2009 Dec 1;9(1):1-12.

19. RCOG -GTG The Investigation and Management of the Small-for-Gestational-Age Fetus Green-top Guideline No. 31,2nd Edition | February 2013 | Minor revisions - January 2014

20. IImpey LWM, Murphy DJ GM. Management of Breech Presentation: Green-top Guideline No. 20b. BJOG An Int J Obstet Gynaecol. 2017;124: e151-e177. 10.1111/1471-0528.1446546

20. Impey LWM, Murphy DJ, Griffiths M, Penna LK on behalf of the Royal College of Obstetricians and Gynaecologists. Management of Breech Presentation. BJOG 2017

; 124: e151-e177.

Impey LWM, Murphy DJ, Griffiths M, Penna LK on behalf of the Royal College of Obstetricians and Gynaecologists. Management of Breech Presentation. BJOG 2017

; 124: e151-e177.

20. lease cite this paper as: Impey LWM, Murphy DJ, Griffiths M, Penna LK on behalf of the Royal College of Obstetricians

and Gynaecologists. Management of Breech Presentation. BJOG 2017

; 124: e151

21. Caradeux J, Martinez-Portilla RJ, Peguero A, Sotiriadis A, Figueras F. Diagnostic performance of third-trimester ultrasound for the prediction of late-onset fetal growth restriction: a systematic review and meta-analysis. YMOB. Elsevier; 2019 May 1;220(5):449-459.e19.

22. Jessie V Madden B, Christopher J Flatley M, Sailesh Kumar. Term small-for-gestational-age infants from low risk women are at significantly greater risk of adverse neonatal outcomes. YMOB. Elsevier Inc; 2018 Feb $17 ;: 1-27$.

23. Lindqvist PG, Molin J. Does antenatal identification of small-for-gestational age fetuses significantly improve their outcome? Ultrasound Obstet Gynecol. 2005 Feb 17;25(3):258-64. 
24. Ciobanu A, Khan N, Syngelaki A, Akolekar R, Nicolaides KH. Routine ultrasound at 32 vs 36 weeks' gestation: prediction of small-for-gestational-age neonates. Ultrasound Obstet Gynecol John Wiley \& Sons, Ltd; 2019 Jun 1;53(6):761-8.

25. Gordijn SJ, Beune IM, Thilaganathan B, Papageorghiou A, Baschat AA, Baker PN, et al. Consensus definition of fetal growth restriction: a Delphi procedure. Ultrasound Obstet Gynecol. John Wiley \& Sons, Ltd; 2016 Sep 1;48(3):333-9.

26. Bricker L, Medley N, Pratt JJ. Routine ultrasound in late pregnancy (after 24 weeks' gestation). Cochrane Pregnancy and Childbirth Group, editor. Cochrane Database of Systematic Reviews John Wiley \& Sons, Ltd; 2015;1(6):1347. Available from: https://www.cochranelibrary.com/cdsr/doi/10.1002/14651858.CD001451.pub4/abstract

27. Henrichs J, Verfaille V, Viester L, Westerneng M, Molewijk B, Franx A, et al. Effectiveness and cost-effectiveness of routine third trimester ultrasound screening for intrauterine growth restriction: study protocol of a nationwide stepped wedge cluster-randomized trial in The Netherlands (The IRIS Study). BMC Pregnancy and Childbirth. BMC Pregnancy and Childbirth; 2016 Oct 12;16(1):1-11.

28. Goldenberg RL, McClure EM. Improving Birth Outcomes in Low- and Middle-Income Countries. BJOG 2017 Dec 14;377(24):2387-8.

29. Ego A, Monier I, Skaare K, Zeitlin J. Antenatal detection of fetal growth restriction and stillbirth risk: a population-based case-control study. Ultrasound Obstet Gynecol. 2019 Jul 31;:uog.20414-34.

30. McClure EM, Saleem S, Goudar SS, Moore JL, Garces A, Esamai F, et al. Stillbirth rates in lowmiddle income countries 2010 - 2013: a population-based, multi-country study from the Global Network. Reproductive Health. BioMed Central Ltd; 2015 Jun 8;12(Suppl 2):S7.

31. Korde-Nayak V. Why is India the world's stillbirth capital: causes and solutions? Banerjee DS, editor. mmj. 2017;1(1):1-14.

32. Singh A, Kumar M. An Analysis of Cause of Stillbirth in a Tertiary Care Hospital of Delhi: A Contribution to the WHO SEARO Project. J Obstet Gynecol India. Springer India; 2019 Apr 1;69(2):155-60.

33. Dandona R, Kumar GA, Kumar A, Singh P, George S, Akbar M, et al. Identification of factors associated with stillbirth in the Indian state of Bihar using verbal autopsy: A population-based study. Bhutta ZA, editor. PLoS Med. 2017 Aug 1;14(8):e1002363-21.

34. Altijani N, Carson C, Choudhury SS, Rani A, Sarma UC, Knight M, et al. Stillbirth among women in nine states in India: rate and risk factors in study of 886,505 women from the annual health survey. BMJ Open. British Medical Journal Publishing Group; 2018 Nov 8;8(11):e022583-13.

35. Dandona R, Kumar GA, Akbar M, Bhattacharya D, Nanda P, Dandona L. Deferred and referred deliveries contribute to stillbirths in the Indian state of Bihar: results from a population-based survey of all births. BMC Med. BioMed Central; 2019 Dec 1;17(1):1-17.

36. Franklin HL, Mirza W, Swanson DL, Newman JE, Goldenberg RL, Muyodi D, et al. Factors influencing referrals for ultrasound- diagnosed complications during prenatal care in five low and middle income countries. Reproductive Health; 2018 Dec 11;:1-9.

37. Stacey T, Thompson JMD, Mitchell EA, Ekeroma A, Zuccollo J, Mccowan LME. Maternal Perception of Fetal Activity and Late Stillbirth Risk: Findings from the Auckland Stillbirth Study. Birth. John Wiley \& Sons, Ltd; 2011 Dec 1;38(4):311-6.

38. Warland J, O'Brien LM, Heazell AEP, Mitchell EA. An international internet survey of the experiences of 1,714 mothers with a late stillbirth: the STARS cohort study. BMC Pregnancy and Childbirth. BioMed Central; 2015 Dec 1;15(1):1-11. 
39. ANM handbook guidelines Available from: https://nhm.gov.in/images/pdf/programmes/maternalhealth/guidelines/sba_guidelines_for_skilled_attendance_at_birth.pdf

40. Winje BA, Wojcieszek AM, Angulo LG, Teoh Z, Norman J, Froen JF, et al. Interventions to enhance maternal awareness of decreased fetal movement: a systematic review. BJOG: An International Journal of Obstetrics and Gynaecology. John Wiley \& Sons, Ltd; 2016 May 1;123(6):886-98.

41. Mangesi L, Hofmeyr GJ, Smith V, Smyth RM. Fetal movement counting for assessment of fetal wellbeing. Cochrane Database of Systematic Reviews. John Wiley \& Sons, Ltd; 2015;(10).

42. Swanson DL, Franklin HL, Swanson JO, Goldenberg RL, McClure EM, Mirza W, et al. Including ultrasound scans in antenatal care in low-resource settings: Considering the complementarity of obstetric ultrasound screening and maternity waiting homes in strengthening referral systems in low-resource, rural settings. Seminars in Perinatology. W.B. Saunders; 2019 Aug 1;43(5):273-81.

Table 1 showing the outcomes of Study group by categories

\begin{tabular}{|c|c|c|c|c|c|}
\hline Outcomes & Category & Risk Ratio & $\begin{array}{l}95 \% \text { Confidence Limits } \\
\text { Lower }\end{array}$ & $\begin{array}{l}95 \% \text { Confidence Limits } \\
\text { Upper }\end{array}$ & $\mathrm{p}$ Value \\
\hline \multirow[t]{3}{*}{ IUFD } & AGA & 1.0 (Ref) & & & \\
\hline & FGR & 2.95 & 0.63 & 13.75 & 0.1494 \\
\hline & SGA & 1.83 & 0.39 & 8.57 & 0.4353 \\
\hline \multirow[t]{3}{*}{ NND $<1$ WEEK } & AGA & 1.0 (Ref) & & & \\
\hline & FGR & 15.75 & 3.56 & 69.58 & $0.0001^{*}$ \\
\hline & SGA & NA & & & \\
\hline \multirow[t]{3}{*}{ NND $>1$ WEEK } & AGA & 1.0 (Ref) & & & \\
\hline & FGR & 9.45 & 2.57 & 34.74 & $0.0001^{*}$ \\
\hline & SGA & 1.47 & 0.17 & 12.49 & 0.7249 \\
\hline \multirow[t]{3}{*}{$\mathrm{NICU}$} & AGA & 1.0 (Ref) & & & \\
\hline & FGR & 5.27 & 2.62 & 10.59 & $0.0001^{*}$ \\
\hline & SGA & 2.78 & 2.75 & 9.35 & $0.0001^{*}$ \\
\hline \multirow[t]{3}{*}{ Ventilation } & AGA & 1.0 (Ref) & & & \\
\hline & FGR & 6.95 & 3.25 & 14.85 & $0.0001 *$ \\
\hline & SGA & 2.59 & 1.03 & 6.48 & $0.0364^{*}$ \\
\hline \multirow[t]{3}{*}{ Composite neonatal morbidity } & AGA & 1.0 (Ref) & & & \\
\hline & FGR & 5.23 & 3.14 & 8.70 & $0.0001^{*}$ \\
\hline & SGA & 2.13 & 1.14 & 4.00 & 0.0183 \\
\hline \multirow[t]{3}{*}{ Severe adverse perinatal outcomes } & AGA & 1.0 (Ref) & & & \\
\hline & FGR & 5.00 & 2.98 & 8.41 & $0.0001^{*}$ \\
\hline & SGA & 1.62 & 0.79 & 3.29 & 0.181 \\
\hline
\end{tabular}

Table 2: Comparison of outcome parameters in the study and the standard group

\begin{tabular}{lllll}
\hline Parameter & Study Cohort & $\begin{array}{l}\text { Non-Study cohort } \\
\text { (Standard Care) }\end{array}$ & $\mathrm{p}$ value & $\begin{array}{l}\text { Relative Risk } \\
\text { (Non-study cohort } \\
\text { vs Study Cohort) }\end{array}$ \\
RR (95\% CI) \\
$\begin{array}{l}\text { Stillbirth rate } \\
\begin{array}{l}\text { per 1000) } \\
\text { NND }<1 \text { week } \\
\text { (per 1000) }\end{array}\end{array}$ & 6.6 & 15.3 & 0.003 & $2.32(1.3-4.13)$ \\
NND $>1$ week & 9.4 & 9.7 & 0.013 & $2.5(1.18-5.34)$ \\
(p 1000$)$ & 9.4 & 0.97 & $1.68(0.89-3.19)$
\end{tabular}




\begin{tabular}{lcccc}
$\begin{array}{l}\text { Perinatal death } \\
\text { rate ( per 1000) }\end{array}$ & 19.9 & 34.4 & 0.0632 & $1.67(0.97-2.89)$ \\
$\begin{array}{l}\text { Intrapartum } \\
\text { LSCS }\end{array}$ & 18.9 & 8.7 & $<0.001$ & $0.46(0.41-0.51)$ \\
$\begin{array}{l}\text { Maternal } \\
\text { mortality (per }\end{array}$ & 60 & 450 & 0.006 & $8.24(1.14-$ \\
\begin{tabular}{l}
$100,000)$ \\
\hline
\end{tabular} & & & & $59.31)$ \\
\hline
\end{tabular}

Table 3(stillbirths ) and Table 4(early and late neonatal deaths)

(uploaded together)

\section{Hosted file}

Table 3 Stillbirths Table 4 Neonatal deaths .docx available at https://authorea.com/users/ 318295/articles/448227-improving-perinatal-outcomes-lessons-learned-from-a-low-middleincome-public-hospital-an-observational-study-from-north-india 